\title{
98.32 Triangles with two angles in the ratio $1: 2$
}

Given $\alpha \in\left(0^{\circ}, 60^{\circ}\right)$, we construct below a triangle $A B C$ with $\gamma=2 \alpha$. We obtain the results of $[1,2]$ by a simpler, shorter and more visual proof.

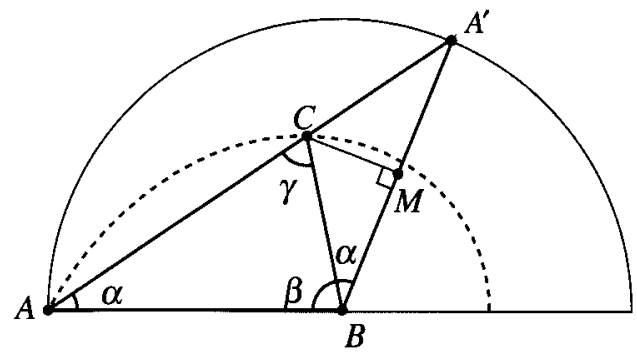

FIGURE 1

In the half-circle centred at $B$ with radius $c=A B$, draw the chord $A A^{\prime}$ with $\angle B A A^{\prime}=\alpha$ and let $C$ be the point where the chord meets the perpendicular bisector of $A^{\prime} B$, so that $\angle A^{\prime} B C=\alpha$. Simple angle chasing shows that $\gamma=2 \alpha$. The similar isosceles triangles $B A^{\prime} C$ and $A^{\prime} A B$ give $\frac{c}{a}=\frac{a+b}{c}$, that is, $c^{2}=a(a+b)$ and $A A^{\prime}=\frac{c^{2}}{a}$. In the right-angled triangle $B M C$ we have $\cos \alpha=\frac{c / 2}{a}$ : the range of $a$ is $(c / 2, c)$ since $\alpha<60^{\circ}$. The bisector $C D$ of $\gamma$ (with foot $D$ on $c$ ) is parallel to $A^{\prime} B$ and of length $\frac{a b}{c}$ by similarity of $A C D$ and $B A^{\prime} C$.

Theorem: The numbers $a, b, c$ are the sides of a triangle with $\gamma=2 \alpha$ for some $\alpha \in\left(0^{\circ}, 60^{\circ}\right)$ if, and only if, $c>0, c / 2<a<c$ and $b=\frac{c^{2}}{a}-a$. Then $\cos \alpha=\frac{c}{2 a}$.

The dashed curve is the locus of $C$ with polar equation $b=c\left(2 \cos \alpha-\frac{1}{2 \cos \alpha}\right)$. The altitude $h_{c}$ attains its maximal length $\frac{1}{2} c \sqrt{6 \sqrt{3}-9} \approx 0.59 c$ for $\alpha=\tan ^{-1}(\sqrt{2 \sqrt{3}-3}) \approx 34.265^{\circ}$. As $\alpha$ increases, $\beta=180^{\circ}-3 \alpha$ decreases. Hence we can describe matters from the perspective of $\beta$. Initially, for 'small' values of $\alpha, \alpha<\gamma<\beta$ with $\beta$ starting from near $180^{\circ}$ and a very flat triangle. The angle $\beta$ reaches $90^{\circ}$ for $\alpha=30^{\circ}, \gamma=60^{\circ}$ and $a=\frac{c}{\sqrt{3}}$. Next we have $\alpha<\beta=\gamma=2 \alpha$ for $\alpha=36^{\circ}$ and $b=c$; this gives us the relation $2 \cos 36^{\circ}-\frac{1}{2 \cos 36^{\circ}}=1$. Thus $2 \cos 36^{\circ}$ is the golden ratio $\phi=\frac{1}{2}(1+\sqrt{5})$ and $\alpha=36^{\circ}$ corresponds to $a=\frac{c}{\phi}$. The next critical moment is when $\alpha=\beta=45^{\circ}$ and, not surprisingly, $a=\frac{c}{\sqrt{ }}$. From that point on we have $\beta<\alpha<\gamma$. As $\alpha$ approaches $60^{\circ}, C$ tends to $A$ and so the triangle collapses with $\beta$ becoming zero and $A B A^{\prime}$ equilateral.

Consider a triangle with $\gamma=2 \alpha$ and integer coprime sides $a$ and $b$ (so that $a$ and $a+b$ are then also coprime). Since $c^{2}=a(a+b), c$ is an integer if, and only if, $a$ and $a+b$ are (coprime) squares: $a=n^{2}$, 
$a+b=m^{2}, b=m^{2}-n^{2}, c=m n$. (If $a, b, c$ are integers with $c^{2}=a(a+b)$, each common divisor of $a$ and $b$ divides $c$.)

Theorem: The integers $a, b, c$ with greatest common divisor 1 are the sides of a triangle with $\gamma=2 \alpha$ if, and only if, $a=n^{2}, b=m^{2}-n^{2}$ and $c=m n$ for coprime integers $n, m$ with $1 \leqslant n<m<2 n$. Then $\cos \alpha=\frac{c}{2 a}=\frac{m}{2 n}$ and the length $\frac{a b}{c}=\frac{n\left(m^{2}-n^{2}\right)}{m}$ of the bisector of $\gamma$ is never an integer.

For example, $n=2, m=3$ gives $a=4, b=5, c=6 ; n=3, m=4$ gives $9,7,12 ; n=3, m=5$ gives $9,16,15$. A heptagonal triangle is defined by angles in the ratio $1: 2: 4$ and is given by the first, second, and fourth vertices of a regular heptagon: it is the unique triangle shape that appears twice in our construction. As $\cos \left(\frac{180^{\circ}}{7}\right) \approx 0.90097$, the triangle with sides 25,56 and 45 (corresponding to $n=5, m=9$ ) is nearly heptagonal.

\section{References}

1. W. W. Willson, A generalisation of a property of the 4, 5, 6 triangle, Math. Gaz. 60 (June 1976) pp. 130-131.

2. R. S. Luthar, Integer-sided triangles with one angle twice another, The College Mathematics Journal 15 (1984) pp. 55-56.

GRÉGOIRE NICOLLIER

University of Applied Sciences of Western Switzerland, Route du Rawyl 47, CH-1950 Sion, Switzerland e-mail: gregoire.nicollier@hevs.ch

\subsection{Computer-generated mathematics: points on the Kiepert hyperbola}

In 1869 Ludwig Kiepert [1] introduced a hyperbola, now known as the Kiepert hyperbola. During the years a number of remarkable points of the triangle have been discovered to lie on the Kiepert hyperbola. In 1994 Eddy and Fritsch discovered that the Spieker centre and the third Brocard point lie on the Kiepert hyperbola ([2, Theorems 3 and 4]). Eric Weisstein [3] has presented a list of 44 points which lie on the Kiepert hyperbola.

We remind the reader of a few definitions. Given $\triangle A B C$ and a point $P$, the complement of the point $P$ is its image under a homothety with centre the centroid $G$ and scale factor $-\frac{1}{2}$. If three lines through the vertices of $\triangle A B C$ are concurrent in a point $P$, then their reflections in the angle bisectors are concurrent in a point called the isogonal conjugate of $P$. Given points $P$ and $Q$, let $P_{A} P_{B} P_{C}$ be the anticevian triangle ${ }^{*}$ of a point $P$ and let $D E F$ be the triangle inscribed in $\triangle A B C$ obtained by $D=P_{A} Q \cap B C$ with similar

$P$ and $P_{A}, P$ and $P_{B}, P$ and $P_{C}$ are harmonic conjugates [4, p. 59] with respect to $A$ and $K, B$ and $L, C$ and $M$ respectively, where $K=A P \cap B C, L=B P \cap C A$ and $M=C P \cap A B$. 